\title{
TWO-STAGE CODE REFERENCE BEAMFORMER IN MOBILE COMMUNICATIONS
}

\author{
Xavier Mestre, Montse Nájar, Miguel A. Lagunas \\ Department of Signal Theory and Communications \\ Universitat Politècnica de Catalunya \\ 08071 BARCELONA, SPAIN \\ e-mail: najar@gps.tsc.upc.es
}

\begin{abstract}
This paper addresses a new architecture for blind adaptive beamforming when dealing with Frequency Hopping (FH) modulation in cellular mobile communications systems. The proposed Code Reference Beamformer (CRB) takes advantage of the inherent frequency diversity to estimate beforehand the noise plus interference correlation matrix, which is employed as the first part of the framework. Then, a second stage is adaptively obtained without any a priori knowledge of either the direction of arrival or the array manifold. Using this information, the first stage is in turn readjusted and, as a result, the scheme is able to track non-stationary scenarios following the channel variations with no previous references.
\end{abstract}

\section{INTRODUCTION}

The performance of antenna arrays in presence of $\mathrm{FH}$ modulation has not been extensively addressed. First, Acar and Compton [1] studied the adverse effects of the FH in a time reference beamformer based on the LMS algorithm. Next, Bakhru and Torrieri [9] proposed an specific algorithm for FH contexts, the Maximin Algorithm, which was based on the spectral characteristics of the received signals. They also studied some techniques of frequency compensation which tried to avoid the algorithm discontinuities at the hop instants. Lastly, Eken [2] addressed a modified sidelobe canceller which needed a priori knowledge of the direction of arrival of the desired signal.

The structure that is proposed in this paper takes advantage of the potential availability of two measurements of the data covariance matrix. This idea has been already used by several authors in non FH contexts. Viberg [10], Souloumiac [7] and Suard [8] proposed other beamforming methods based on the estimation of two different covariance matrices. However, it has been shown [3] that the gradient-like adaptive algorithm described in this paper presents a higher convergence rate and performs better in presence of errors in the estimated matrices.

\section{TWO-STAGE CODE REFERENCE BEAMFORMER}

It is well know that the maximum Signal to Interference plus Noise Ratio (SINR) solution for a beamformer is proportional to

This work was partially supported by the National Research Plan of Spain, CICYT, TIC 95-1022-C05-1, TIC96-0500-C1001 and by the Generalitat de Catalunya, CIRIT, 1996GR-0096 the product of the inverse of the interference plus noise covariance matrix and the steering vector of the desired signal:

$$
\mathbf{u}_{\text {opt }}=\mu \mathbf{R}_{n}^{-1} \mathbf{s}_{d}
$$

The optimal beamvector can be implemented using two different stages corresponding to the two factors in (1). The first one will consist of a preprocessing of the incoming snapshot by the inverse of the interference plus noise correlation matrix $\mathbf{R}_{n}{ }^{-1}$, while the second one will be the steering vector of the signal of interest. It can be proved that in scenarios with high interference power level, the first stage acts as a blocking matrix for the interfering space. This stage will be therefore devoted to forming deep nulls in the directions of arrival of the interfering signals, whereas the second one will steer the main beam towards the direction of arrival of the desired signal.

The proposed system [3-6] is outlined in Figure 1. The framework takes advantage of the Frequency Hopping Modulation working with two parallel processors. One of them, named the anticipative processor, is considered for estimating the noise plus interference covariance matrix. The idea is to obtain a previous image of the scenario down-converting the received signal with the carrier frequency of the next hop $f_{i+1}$. Thus, the first stage of the optimum beamformer for the following frequency is calculated beforehand.

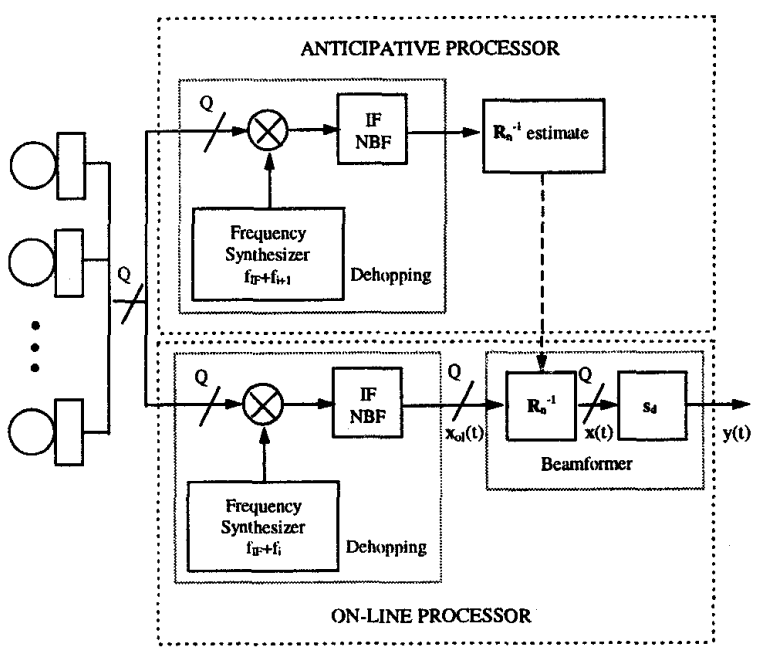

Figure 1. Code Reference Beamformer 
When a frequency hop occurs, the estimated matrix is transferred to the on-line processor. The second stage is not always available, since in a great number of applications -such as mobile communications- the angle of arrival of the desired signal is unknown. This implies that a blind estimation of the second stage is needed; hence, the adaptive array problem is to choose the weight vector $\mathbf{w}$ corresponding to the second stage following the maximum SINR criterion:

$$
\left.\operatorname{SINR}\right|_{\max }=\frac{\mathbf{w}^{H} \mathbf{R}_{x} \mathbf{w}}{\mathbf{w}^{H} \mathbf{R}_{n}^{-1} \mathbf{w}}-\left.1\right|_{\max }
$$

where $\mathbf{R}_{\mathrm{x}}$ is the correlation matrix of the output of the first stage and $\mathbf{w}$ the weight vector to be estimated. The problem stated in (2) can be formulated as a constrained maximization of the array output power:

$$
\begin{gathered}
\left.\mathbf{w}^{\mathrm{H}} \mathbf{R}_{\mathbf{x}} \mathbf{w}\right|_{\max } \\
\text { subject to } \mathbf{w}^{\mathrm{H}} \mathbf{R}_{\mathbf{n}}^{-1} \mathbf{w}=1
\end{gathered}
$$

It can be easily proved that the solution to (3) is the generalized eigenvector of the pencil $\left(\mathbf{R}_{x}, \mathbf{R}_{n}^{-1}\right)$ corresponding to the generalized maximum eigenvalue:

$$
\mathbf{R}_{x} \mathbf{w}_{\text {opt }}=\lambda_{\max } \mathbf{R}_{n}^{-1} \mathbf{w}_{\text {opt }}
$$

being the generalized maximum eigenvalue $\lambda_{\max }$ equal to the array output power:

$$
\lambda_{\max }=\mathbf{w}_{o p t}^{H} \mathbf{R}_{x} \mathbf{w}_{o p t}
$$

The cost function utilized to develop the gradient search adaptive algorithm is formulated as the Lagrangian defined from (3):

$$
L=\mathbf{w}^{H} \mathbf{R}_{x} \mathbf{w}-\lambda_{\max }\left(\mathbf{w}^{H} \mathbf{R}_{n}^{-1} \mathbf{w}-1\right)
$$

We derive the suggested adaptation rule calculating the gradient respect to $w^{\mathrm{H}}$ of the above expression:

$$
\mathbf{w}(n+1)=\mathbf{w}(n)+\mu\left(\mathbf{R}_{x}-\lambda_{\max } \mathbf{R}_{n}^{-1}\right) \mathbf{w}(n)
$$

with $\mu$ a parameter that controls both the convergence rate and the stability of the algorithm. The computational cost is finally reduced substituting the first term of the Lagrangian gradient by its instantaneous estimate:

$$
\mathbf{w}(n+1)=\mathbf{w}(n)+\frac{\alpha / \delta_{\max }}{(\lambda(n)-1)}\left[\mathbf{x}(n) y^{*}(n)-\lambda(n) \mathbf{R}_{n}^{-1} \mathbf{w}(n)\right]
$$

being $\delta_{\max }$ the maximum eigenvalue of the matrix $\mathbf{R}_{n}{ }^{-1}$, namely the inverse of the noise power. Note that the parameter $\mu$ in (7) has been normalized, so the trade-off between convergence rate and misadjustment will depend upon the constant $\alpha$. The generalized maximum eigenvalue is calculated as the array output power:

$$
\lambda(n+1)=\beta \lambda(n)+(1-\beta)|y(n+1)|^{2}
$$

It can be shown [6] that the procedure yields the generalized eigenvector corresponding to the generalized maximum eigenvalue of the pencil $\left(\mathbf{R}_{\mathrm{x}}, \mathbf{R}_{\mathrm{n}}{ }^{-1}\right)$. Nevertheless, when the power of the interferences impinging on the array approaches infinity, the algorithm converges to [3]:

$$
\mathbf{w}(n)=\mathbf{Q}, \mathbf{Q}_{s}^{H} \mathbf{w}(0)
$$

where $\mathbf{Q}_{\mathrm{s}}$ is the unitary matrix whose columns span the total signal subspace, including desired and interfering components. Noting that $\mathbf{Q}_{s}{ }^{\mathrm{H}} \mathbf{Q}_{\mathrm{s}}=\mathbf{I}$, it turns out that the steady state solution described by $(10)$ is the projection of the initialization vector onto the signal subspace. Thereby, the beamvector corresponding to the second stage of the on-line processor will actually depend on the initialization of the algorithm. Recalling, however, that the first stage is orthogonal to the interfering subspace, the complete beamformer becomes identical to the optimum solution up to a scale factor:

$$
\mathbf{u}(n)=\mathbf{R}_{n}^{-1} \mathbf{Q}_{s} \mathbf{Q}_{s}^{H} \mathbf{w}(0)=\mathbf{R}_{n}^{-1} \mathbf{w}_{\text {opt }} \mathbf{w}_{\text {opt }}^{H} \mathbf{w}(0)=k \mathbf{R}_{n}^{-1} \mathbf{s}_{d}
$$

and should therefore yield identical performance. The system will consequently exhibit a rapid convergence rate even in presence of high power interferences.

When a new frequency hop occurs, the estimated generalized eigenvector should be modified in order to adapt the second stage for a new frequency reception. If the power of the interferences is not extremely high, the estimation of the generalized eigenvector will converge to the steering vector of the signal of interest $s_{\mathrm{d}}$. Hence, the phases of the weights will be linearly dependent on the frequency utilized (module $2 \pi$ ) provided that no phase calibration errors are present. The socalled frequency focusing technique will consist in a simple phase multiplication by the frequency ratio $f_{i+1} / f_{i}$. This method will provide a good estimation of the optimum beamvector for the following frequency, reducing the characteristic SINR falls at the hop instants. In any case, simulations reveal that frequency focusing improves the beamformer performance even in situations with high interfering power and in presence of phase calibration errors.

\section{GENERALISATION TO MOBILE RADIO COMMUNICATION SCENARIOS}

In mobile communications systems, wave reflections cause a single emitter to contribute several correlated wave fronts to the field illuminating the array. This phenomenon, along with the presence of doppler, will originate the characteristic fading effects on the received signals. In this paper, the delay spread of the mobile channel is assumed to be much lower than the inverse of the symbol rate, so the channel frequency selectivity is disregarded. Under this condition, the solution that maximizes the SINR at the array output is still (1), although now $s_{\mathrm{d}}$ stands for a generalized stecring vector that depends on time due to the channel fading:

$$
\mathbf{s}_{d}(n)=\frac{1}{N} \sum_{i=1}^{N} a_{i}(n) \mathbf{s}_{d t}
$$

with $\mathrm{N}$ the number of propagation paths, $\mathrm{a}_{\mathrm{i}}(\mathrm{n})$ the reflection coefficient for path $i$, and $s_{d i}$ the steering vector for the corresponding moving source. This composite nature of $\mathbf{s}_{\mathrm{d}}(\mathrm{n})$ does not affect the CRB behavior, since a blind approach has been considered for its estimation. 
Due to the presence of fading on the received interferences, the first stage estimated by the anticipative processor should be continuously modified in order

to track the non-stationary scenario. The solution that is put forward has been depicted in Figure 2.

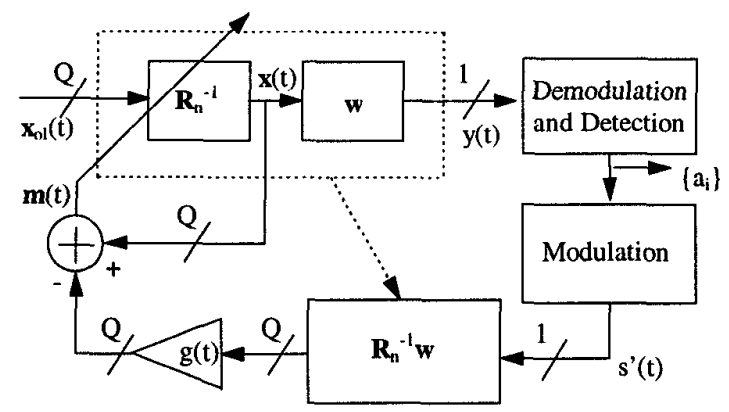

Figure 2. Adaptation of the first stage.

The inverse correlation matrix estimated by the anticipative processor is adequate only as an initialization of the system. Once it has been transferred to the on-line processor and after a few iterations aimed to achieve global convergence, the first stage is continuously updated using the following equation:

$$
\mathbf{R}_{n}^{-1}(n+1)=\gamma \mathbf{R}_{n}^{-1}(n)+(1-\gamma) \mathbf{m}(n+1) \mathbf{m}^{H}(n+1)
$$

with $\mathbf{m}(\mathrm{n})$ an estimation of the undesired component of the incoming snapshot preprocessed by the first stage of the beamformer. If a replica of the desired signal $s(n)$ and the equivalent steering vector $s_{d}$ were available, $m(n)$ could be calculated as:

$$
\mathbf{m}_{o p t}(n)=\mathbf{x}(n)-s(n) \mathbf{R}_{n}^{-1} \mathbf{s}_{d}
$$

Nevertheless, the desired signal power is always unknown, as well as the generalized steering vector $s_{d}$ in presence of high power interferences. Note, however, that a noiseless regenerated signal $s^{\prime}(n)$ can be extracted from the beamformer output $y(n)$, even though it is not free of the possible detection errors. Fortunately, all these drawbacks can be easily overcome by means of an adaptive coefficient $\mathrm{g}(\mathrm{n})$ continuously adjusted to minimize the norm of the vector $\mathbf{m}(\mathrm{n})$ :

$$
\mathbf{m}(n)=\mathbf{x}(n)-g(n) s^{\prime}(n) \mathbf{R}_{n}^{-1} \mathbf{w}
$$

Recall now from (11) that, once convergence occurs, the global response of the complete beamformer is equal to the optimum one up to a scalar factor. Hence, the adaptive coefficient $g(n)$ will be automatically adjusted so that the vector $\mathbf{m}(n)$ will converge to the value indicated in (14). We finally stress that, thanks to this coefficient, the first stage will be properly adapted even in presence of high power interferences or detection errors. The described procedure does not make use of any matrix inversions, so it will be able to operate without numerical problems in scenarios with high power interferents, where the matrix $\mathbf{R}_{n}^{-1}$ may be ill-conditioned.

It is accepted that in frequency jumping systems the frequency spacing is always set higher than the coherence bandwidth of the mobile channel. Therefore, two consecutive channels will appear as statistically independent, and the same will happen to the generalized steering vectors $\mathbf{s}_{\mathrm{d}}(\mathrm{n})$. This fact, along with the lack of linear dependence on the frequency of the equivalent steering vector elements, might contribute to a degradation of the results achieved with the frequency focusing process. Nevertheless, results show that it is still convenient to focus the second stage at the hop instants, since there is always spatial information that will be taken into account in order to obtain a proper initialization for the next hop.

\section{SIMULATIONS}

In this section, we study the performance of the new beamformer proposed in this paper. The simulations have been made with a linear equally spaced array of 4 sensors in which the interelement separation was half a wavelength at the center frequency of the hopping band $(900 \mathrm{MHz})$. All the trasmitted signals were spread uniformly over a 50 per cent relative bandwidth, which is the ratio of the total hopping bandwidth to the center frequency.

A mobile radio scenario with three users was considered. All the stations transmitted GMSK signals (4 samples/symbol) with a normalized $3 \mathrm{~dB}$ cut-off frequency of the gaussian filter equal to 0.3 . The array was illuminated from elevations of 20 (desired signal), -30 and 40 degrees (interfering signals) and the received $\mathrm{Eb} / \mathrm{No}$ was equal to $15 \mathrm{~dB}$ for the three sources. All the permitted frequencies of the system were simultaneously used by the interfering mobiles. Independent Rayleigh channels were generated assuming a speed of the users of $280 \mathrm{Km} / \mathrm{h}$ in the direction of the array broadside; a maximum Doppler frequency of $+/-233 \mathrm{~Hz}$ was therefore considered. The sampling rate was assumed to be $1.11 \mathrm{Msamples} / \mathrm{seg}$ and the dwell time or duration of the hop interval was set to the duration of 150 symbols (which is similar to the number of symbols in a burst of the GSM system). A random sequence of 1200 symbols was generated, so 8 frequency hops occurred. Note that, with the above specifications, the simulated bit rate was $277 \mathrm{Kbits} / \mathrm{seg}$., which is approximately the transmission bit rate of the GSM system. Concerning the adaptation of the first stage, the coefficient $g(n)$ was updated using a LMS algorithm and the received signal was incoherently demodulated by means of a differential detector.

First, the case of low angular dispersion of the impinging sources was considered (note that this is an appropriate model for rural scenarios). In the simulation, each user contributed 50 rays with random directions of arrival, which were generated with a gaussian model within a beamwidth of 15 degrees. In Figure 3, the SINR and the beamvector evolutions have been depicted with solid line, while the optimum values have been represented using a dotted one. The fast convergence of the proposed beamformer and the reduction of SINR falls achieved with frequency focusing are illustrated.

Next, in order to consider indoor and urban scenarios, attention is turned to the case of high angular dispersion of the users' spatial signature. Thus, the directions of arrival were randomly generated using a uniform distribution model. The SINR evolution has been plot with solid line in Figure 4, along with its optimum values (dotted line). It can be noticed that the two-stage CRB is able to follow the channel variations without any a priori reference. However this tracking ability is achieved at the expense of misadjustment. The SINR falls at the hop instants can 
not be avoided, since no spatial information of the desired user can be re-utilized.
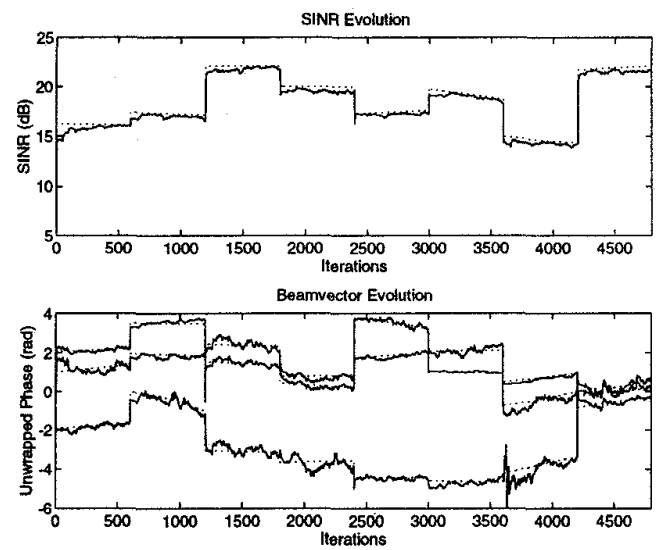

Figure 3. Case of low angular dispersion of the impinging sources (rural scenarios). Each user contributed 50 rays with random directions of arrival, which were generated with a gaussian model within a beamwidth of 15 degrees. Solid line: estimated values. Dotted line: optimum values.

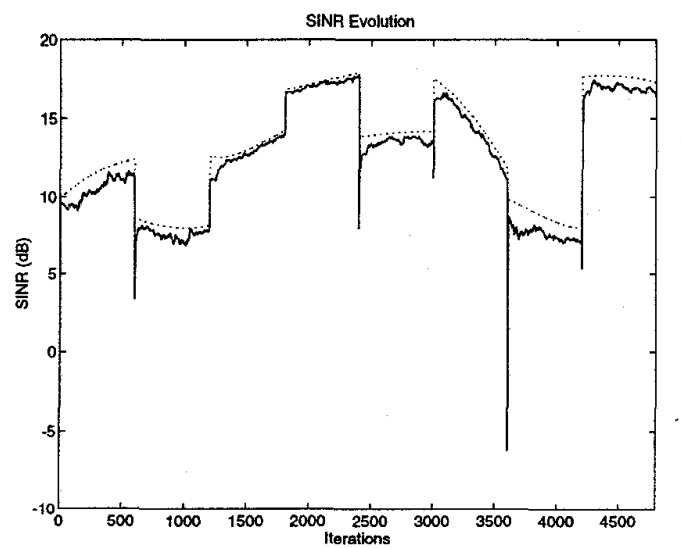

Figure 4. Case of high angular dispersion of the users' spatial signature (indoor and urban scenarios). The directions of arrival were randomly generated using a uniform distribution model. Solid line: estimated values. Dotted line: optimum values.

\section{CONCLUSIONS}

A new method for beamforming in FH modulation contexts has been presented. It has been shown that the proposed framework does not need any knowledge of either the direction of arrival of the desired signal or the array manifold. Furthermore, the system is able to follow the channel variations in a mobile communications environment, tracking non-stationary scenarios without any additional information. Finally, it has been shown that frequency focusing of the second stage reduces the SINR falls at the hop instants even in adverse situations like mobile communications. To recap, high tracking capability, reduction of the SINR falls and robustness to calibration errors are the main features of the proposed beamformer.

\section{REFERENCES}

[1] Acar L., Compton R., "The Performance of an LMS Adaptive Array with Frequency Hopped Signals", IEEE Transactions on Aerospace and Electronic Systems, vol. AES-21, no.3, pp. 360-371, May 1985.

[2] Eken F., "Use of Antenna Nulling with Frequency Hopping Against the Follower Jammer", IEEE Transactions on Antennas and Propagation, vol. AP-39, no.9, pp. 13911397, September 1991.

[3] Nájar M., "Conformador de haz de referencia por código de dos etapas: aplicación a la recepción de señales con modulación de saltos frecuenciales", $\mathrm{PhD}$ dissertation, October 1996.

[4] Nájar M., Lagunas M. A., "High Resolution Adaptive Arrays Based on Random Processing Techniques: Frequency Hopping Modulation", Proceedings of the IEEE International Conference on Acoustics, Speech and Signal Processing ICASSP-95, pp. 1737-1740, Detroit, May 8-12, 1995.

[5] Nájar M., Lagunas M. A., "Adaptive Array Beamforming for Frequency Hopping Modulation", Proceedings of the VIII European Signal Processing Conference EUSIPCO-96, vol. 2, pp. 939-942, Trieste, September 10-13, 1996.

[6] Nájar M., Lagunas M. A., "Two-Stage Code Refernce Beamformer for the reception of frequency hopping modulated signals", submitted to the IEEE Transactions on Aerospace and Electronic Systems, en proceso de revisión.

[7] Souloumiac A., "Blind Source Detection and Separation Using Second Order Non-Stationarity", Proceedings of the IEEE International Conference on Acoustics, Speech and Signal Processing ICASSP-95, pp. 1912-1915, Detroit, May 8-12, 1995.

[8] Suard B., Naguib A. F., Xu G., Paulraj A., "Performance of CDMA Mobile Communication Systems Using Antenna Arrays", Proceedings of the IEEE International Conference on Acoustics, Speech and Signal Processing ICASSP-93, pp. IV153-IV156, Minneapolis, April 27-30, 1993.

[9] Torrieri D., Bakhru K., "Frequency Compensation in an Adaptive Antenna System for Frequency-Hopping Communications", IEEE Transactions on Aerospace and Electronic Systems, vol. AES-23, no. 4, pp. 448-467, July 1987.

[10] Viberg M., "Sensor Array Processing Using Gated Signals", IEEE Transactions on Acoustics, Speech and Signal Processing, vol. ASSP-37, no. 3, pp. 447-450, March 1989. 